\title{
Reversión a la media en las series de precios reales del petróleo en México
}

\author{
Mean reversion in Mexico's real oil price series \\ José Carlos Ramírez Sánchez ${ }^{*}$, Fernando Cruz Aranda ${ }^{2}$ \\ Agustín Cabrera LLanos ${ }^{3}$ \\ ${ }^{1}$ Profesor-investigador del Departamento de Estudios Economicos \\ de El Colegio de la Frontera Norte, Tijuana, Mx. \\ ${ }^{2}$ Universidad Panamericana, México \\ ${ }^{3}$ Unidad Profesional Interdisciplinaria de Biotecnología del Instituto Politécnico Nacional, México
}

Recibido el 7 de febrero de 2019; aceptado el 4 de junio de 2019

Disponible en Internet el: 4 de junio de 2019

\section{Resumen}

El objetivo de este documento es mostrar la existencia de un patrón de reversión a la media en la serie de precios reales del petróleo exportado por México al continente Americano entre enero de 1999 y junio de 2017. Con ese fin adaptamos una ecuación en diferencias estocástica a la serie de precios de la variedad Maya para hacer pronósticos dentro y fuera de la muestra, con una ventana de seis y doce meses. Los principales resultados obtenidos muestran que, en efecto, hay una reversión a la media de largo plazo en los precios inicialmente supuestos como racionales. Otras pruebas estadísticas confirman que esta reversión a la media es persistente en virtud de que los shocks producidos sobre los precios reales no involucran cambios permanentes.

Código JEL: C01, C13, C65, G12, G23

Palabras clave: Precios de petróleo de México; Reversión a la media; Hipótesis de mercados eficientes; Funciones de impulso respuesta; Pronóstico de precios

\footnotetext{
*Autor para correspondencia

Correo electrónico jcramirez@colef.mx (J.Ramírez).

La revisión por pares es responsabilidad de la Universidad Nacional Autónoma de México. 


\begin{abstract}
This paper aims at showing the existence of a mean reversion pattern in the series of real prices of oil exported by Mexico to the American Continent between January 1999 and June 2017. For this purpose we adapt a stochastic difference-equation to the series of prices of Maya variety to make forecasts in-and out of the sample, with a window of six and twelve months. The main results drawn from the best-fit model show that, in effect, there is a reversion to the long-term mean in prices initially assumed to be rational. Other statistical tests confirm that this reversion to the mean is persistent because the shocks produced on real prices do not involve permanent changes.
\end{abstract}

JEL codes: $\mathrm{C} 01, \mathrm{C} 13, \mathrm{C} 65, \mathrm{G} 12, \mathrm{G} 23$

Keywords: Mexico's oil-prices; Mean reversion; Efficient-market hypothesis; Impulse-response functions; Price forecasting

\title{
Introducción
}

La hipótesis de que los precios del petróleo crudo siguen una caminata aleatoria sin deriva (drift) es un punto de referencia para cualquier pronóstico. Su rechazo o aceptación decide, prácticamente, la utilidad del ejercicio predictivo, pues sin su prueba el modelo empleado estaría en entredicho. Por eso es que para entender el comportamiento de los precios del crudo, resulta importante conocer, antes, las razones detrás de la adopción de esa hipótesis. Y, para tal efecto, conviene dividir esas razones en teóricas y estadísticas. Entre las primeras razones destacan dos que están relacionadas con las condiciones de equilibrio derivadas de la posibilidad de almacenamiento del crudo y de la contratación de futuros por parte de los agentes (Hamilton, 2009).

Respecto a la posibilidad de almacenamiento, el productor siempre estará forzado a cumplir la condición (1) en la que se establece que el valor esperado del precio futuro $E\left(P_{t+1}\right)$ es igual al precio spot $P_{t}$ más el valor presente del costo neto de acarreo o de cualquier otra prima sobre el riesgo $C_{t}$, que induzca a los inversionista a mantener mayores o menores niveles de inventarios de crudo.

$$
E\left(P_{t+1}\right)=P_{t}+C_{t}
$$

En caso que se pierda el equilibrio en (1), la desigualdad tenderá a ser corregida una vez que las acciones de los agentes, guiadas por las expectativas sobre $P_{t+1}$, reflejen los ajustes en $P_{t}$. De esta manera tenemos, por ejemplo, que si los inversionistas creen que $E\left(P_{t+1}\right)>P_{t}+C_{t}\left(E\left(P_{t+1}\right)<P_{t}+C_{t}\right)$ ellos buscarán comprar (vender) mayores volúmenes de crudo 
para almacenarlo (liberarlo) y venderlo en el futuro (presente), con el consecuente incremento (descenso) en el precio spot.

Una situación similar se presenta cuando existe la posibilidad de contratar un futuro $F_{t}$ para adquirir cierto volumen de crudo un año después. Si las expectativas del precio del petróleo son tales que $E\left(P_{t+1}\right)>F_{t}\left(E\left(P_{t+1}\right)<F_{t}\right)$ entonces es claro que los agentes encontrarán más rentable contratar (no contratar) futuros y esperar un año para iniciar (comenzar ahora) operaciones. Ante la mayor (menor) demanda de futuros, el valor de $F_{t}$ aumentará (disminuirá) de tal suerte que el equilibrio de (2) se restablecerá en el presente.

$$
F_{t}=E\left(P_{t+1}\right)+R_{t}
$$

donde $R_{t}$ es el valor esperado de cualquier prima de riesgo o costo de transacción.

Los equilibrios en (1) y (2) no son más que dos formas distintas de parafrasear la hipótesis débil de mercado eficiente (HME), según la cual los precios del crudo describen una caminata aleatoria con incrementos independientes y cuyas predicciones en el tiempo $t+1$ dependen enteramente de la información que fluya en ese periodo. Por eso es que no hay posibilidad de arbitraje con el uso de la información del periodo $t$, a menos que existan imperfecciones en el mercado.

Para ver las implicaciones de la HME más formalmente consideremos que los componentes distintos a $P_{t}$ y $F_{t}$ en (1) y (2) representan, en ambos casos, los precios racionales ex post $P_{t}^{*}$ o precios que incluyen el valor presente descontado de las primas de riesgo y de los costos de transacción; esto es que:

$$
P_{t}^{*}=E\left(P_{t+1}\right)-C_{t}=E\left(P_{t+1}\right)+R_{t}
$$

De acuerdo con la HME, los precios reales $P_{t}$ y $F_{t}$ constituyen el mejor pronóstico de los precios racionales ex post y cualquier movimiento sorpresivo en el mercado petrolero dependerá exclusivamente de $P_{t}^{*}$. Pero, debido a que es imposible que siempre se cumpla la condición $P_{t}^{*}=P_{t}$ a lo largo del periodo de tiempo, por la presencia de desviaciones aleatorias, es más factible esperar que:

$$
P_{t}^{*}=P_{t}+u_{t}
$$

donde $u_{t}$ es el error de pronóstico, ocasionado por la expedición de deuda o recompra de empresas, entre otras explicaciones.

Ahora bien, debido al supuesto de incrementos independientes de la caminata aleatoria, $u_{t}$ no puede estar correlacionada con la información disponible en $t$ ni con $P_{t}$, por lo que la $\operatorname{Var}\left(P_{t}^{*}\right)=\operatorname{Var}\left(P_{t}\right)+\operatorname{Var}\left(u_{t}\right)$. De aquí que la máxima varianza posible de un pronóstico óptimo no puede ser mayor a la varianza de la variable pronosticada y, como consecuencia, las volatilidades de las series de precios racionales y reales deben estar contenidas dentro de 
ciertos límites (Shiller, 2003) definidos por:

$$
\operatorname{Var}\left(P_{t}\right) \leq \operatorname{Var}\left(P_{t}^{*}\right)
$$

Las pruebas empíricas de (4) y (5) han producido una cantidad irregular de literatura que, a grandes rasgos, se divide en dos vertientes. Y aquí entramos a las razones estadísticas. La primera vertiente sostiene que, en efecto, los precios reales siguen una caminata aleatoria sin drift y, por tanto, son impronosticables con la información pasada, ya sea que ésta se exprese en niveles o en tasas de crecimiento (Hamilton, 2009). La fundamentación radica en el hecho de que $u_{t}$ crecerá a una tasa $\mathrm{S} \sqrt{t}$, que es precisamente la desviación estándar de una caminata aleatoria, en cualquier simulación diseñada para predecir los precios racionales con base en los precios reales pasados.

La segunda vertiente considera, en cambio, que los precios del petróleo crudo tienen otros determinantes, distintos de $C_{t}$ y $R_{t}$, que hacen posible su pronóstico. Entre estos determinantes destacan las elasticidades de los precios de los productos petrolíferos y del ingreso de los consumidores, las políticas de abastecimiento y fijación de bandas de precios de los países productores, la renta por escasez del crudo, el agotamiento de los principales yacimientos y la emergencia de nuevos consumidores (Hamilton, 2009). Adicionalmente, se reconocen como causantes de las fluctuaciones del precio del petróleo a: i) los cambios en la oferta de hidrocarburos debido a eventos políticos, nuevos descubrimientos y mejoras tecnológicas en el proceso de extracción; y a ii) los cambios abruptos en la demanda o en las expectativas sobre el ritmo futuro de la oferta de petróleo (Baumeister y Lilian, 2016). Cada factor tiene, obviamente, un peso distinto según sean las regiones y períodos considerados.

La base teórica de los pronósticos descansa, precisamente, en el hecho de que esos determinantes desvían a los precios reales del petróleo de sus precios racionales en forma temporal (reversión a la media) o permanente (cambio de régimen). La búsqueda de patrones estadísticos de ese alejamiento incluye métodos que capturan relaciones lineales, como los modelos ARIMA, GARCH y ECM, y no lineales, como los modelos de inteligencia artificial o de redes neuronales, entre los precios del crudo y las variables asociadas con los determinantes (Safari y Davallou, 2018). Los resultados arrojan evidencia mixta que apoyan la existencia de ambos tipos de patrones.

Este trabajo se inscribe en la segunda vertiente y tiene como objetivo mostrar que la serie de precios de petróleo de la modalidad Maya en México exhibe reversión a la media en el largo plazo. Es decir, que las volatilidades son tales que violan significativamente los límites de la ecuación (5) y, por tanto, permiten asociarle a los precios del crudo un patrón de comportamiento pronosticable en el corto plazo. Los resultados obtenidos con la simulación del modelo y la prueba de Andrews sobre estacionariedad revelan que la reversión a la media de las series de precios comprendidas entre 1999 y 2017 es persistente y sin cambios permanentes en sus tendencias. 
El documento consta de dos secciones adicionales. La segunda presenta una breve revisión de la literatura, seguida de la derivación de la ecuación en diferencia estocástica de precios y del análisis estadístico de la base de datos. La tercera sección presenta la simulación y las pruebas para analizar los errores de pronóstico de los ajustes seleccionados. Las conclusiones resumen los principales resultados del trabajo.

\section{Breve revisión de la literatura y metodología empleada}

En contraste con la abundante literatura relacionada con las técnicas de pronóstico, hay relativamente pocos estudios que investiguen los patrones de reversión a la media en los precios reales del petróleo crudo. Las razones parecen residir en la marcada asociación de estos patrones con el comportamiento de variables como las tasas de interés y al predominio abrumador de artículos que privilegian el uso de nuevos métodos heurísticos e híbridos en el pronóstico.

En cualquier caso los artículos existentes muestran evidencia de reversión a la media en casos específicos, como es en la política de banda de precios utilizada por la OPEP en su estrategia de manejo de la oferta de petróleo (Hammoudeh, 1996) y en casos generales, como es en las series de precios de 44 commodities utilizadas por el Banco Mundial para varios países entre 1958 y 1997 (Cashin et al., 2000). En esos artículos se confirma el hecho ya probado por otros autores de que los shocks sobre los precios del petróleo son de larga duración y que, pasado un tiempo, las series recuperan su nivel original (véase a Choi y Hammoudeh. 2009; Li y Thompson, 2010). Es decir, se confirma que las series de precios del petróleo no son procesos de raíz unitaria y que los shocks pueden desplazar los precios hasta más de 100 unidades y recuperar la mitad de su nivel original en 3 años (Cashin et al., 2000).

En México, la mayor parte de la literatura está concentrada en analizar los efectos de las volatilidades de los precios del crudo sobre el tipo de cambio o el funcionamiento del mercado de valores, pero no en investigar patrones de reversión a la media (véase, por ejemplo, Bermúdez et al., 2018; Valdés et al., 2016). Para subsanar este vacío desarrollamos una metodología que incluye dos etapas sucesivas. La primera consiste en proponer una ecuación dinámica de los precios reales con base en el modelo de Cox, Ingersall y Ross (CIR, 1985). La segunda se aboca a simular dicha ecuación usando la serie de precios reales de la variedad Maya exportada al continente Americano.

\section{El modelo de reversión a la media}

En lo que toca a la primera etapa, cabe señalar que optamos por derivar una ecuación estocástica del modelo CIR, porque presenta algunas ventajas para el análisis de las series de tiempo del petróleo, tales como su condición de no negatividad (no garantizada, por ejemplo, 
en Vasicek, 1977) y su ductilidad en el proceso de discretización de los parámetros de los precios. Ambas características hacen de la ecuación un medio ideal para detectar parones de reversión a la media en modelos auto-regresivos, cuya simulación requiere del cómputo de medidas de persistencia. En concreto suponemos que la dinámica de los precios está gobernada inicialmente por (6)

$$
\mathrm{d} P_{t, x}=a_{x}\left(b_{x}-P_{t, x}\right) \mathrm{d} t+\sigma_{x} \sqrt{P_{t, x}} \mathrm{~d} W_{x, t}
$$

donde $P_{t, x}$ es el precio del petróleo crudo al tiempo $t, a_{x}$ es la velocidad de ajuste de los precios de crudo para $\mathrm{x}=$ variedad Maya, $\left(b_{x}-P_{t, x}\right)$ es la corrección hacia la media en el largo plazo, $\sigma_{x}$ es la desviación estándar de los precios, $t=t_{1}, t_{2}, t_{3}, \ldots, t_{n_{c}}$ los periodos de tiempo considerados; $\left\{W_{x}\right\}_{t \geq 0}$ es un movimiento Browniano definido sobre un espacio fijo de probabilidad $(\Omega, \mathcal{F}, \mathbb{P})$ en la que $\Omega$ es el espacio muestral, $\mathcal{F}$ una sigma algebra y $\mathbb{P}$ la medida de probabilidad.

Debido a que en su estado actual (6) es de orden muy general y poco útil para cualquier simulación es necesario discretizarla, antes, para poder tratarla con métodos estadísticos. Así que para calcular adecuadamente los valores de los parámetros de interés, $\left(a_{x}, b_{x} \mathrm{y} \sigma_{x}{ }^{2}\right)$ en una serie de tiempo, conviene aplicar el siguiente cambio de variable $y_{t ; x}=y\left(P_{t ; x}, t\right)=2\left[P_{t ; x}\right]^{1 / 2}$.. De este modo, las derivadas de primero y segundo ordenes de $y_{t ; x}$ con respecto a $P_{t ; x}$, toman la siguiente forma:

$$
\frac{\partial y_{t ; x}}{\partial P_{t, x}}=\frac{1}{P_{t, x}}=\frac{2}{y_{t ; x}}
$$

$\mathrm{y}$

$$
\frac{\partial^{2} y_{t ; x}}{\partial P_{t, x}^{2}}=-\frac{1}{2 P_{t, x} \sqrt{P_{t ; x}}}=-\frac{1}{P_{t ; x} y_{t ; x}}
$$

Una vez aplicado el lema de Itô se obtiene $\mathrm{d} y_{t, x}$ como una ecuación diferencial estocástica con varianza constante; esto es:

$$
\mathrm{d} y_{t, x}=\left[\left(2 a_{x} b_{x}-\frac{\sigma_{x}^{2}}{2}\right) \frac{1}{y_{t ; x}}-\left(1-\frac{a_{x}}{2}\right) y_{t ; x}\right] \mathrm{d} t+\sigma_{x} \mathrm{~d} W_{x, t}
$$


Cuya expresión discreta es de la forma:

$$
\begin{aligned}
& y_{t+1, x}=\beta_{1 ; x}\left[\frac{1}{y_{t ; x}}\right]+\beta_{2 ; x} y_{t ; x}+\varepsilon_{t} . \\
& \operatorname{con} \beta_{1 ; x}=2 a_{x} b_{x}-\frac{\sigma_{x}^{2}}{2}, \beta_{2 ; x}=1-\frac{a_{x}}{2}, x=0,1, \ldots \mathrm{y} \\
& t=t_{1}, t_{2}, t_{3}, \ldots, t_{n_{c}} .
\end{aligned}
$$

Tras algunas manipulaciones se llega a que $\widehat{a_{x}}=2\left(1-\widehat{\beta_{2}}\right)$ y $\widehat{b_{x}}=\frac{\sigma_{x}^{2}+2 \widehat{\beta_{1}}}{8\left(4-\widehat{\beta_{2}}\right)}$. Con estos resultados se tiene, finalmente, que el precio estimado está dado por:

$$
\hat{P}(x, t)=\left(\frac{\widehat{y_{x}}}{2}\right)^{2} \text {. }
$$

Las ecuaciones (8) y (9) son, ahora sí, adecuadas para nuestros fines debido a que la estimación de sus parámetros nos permite analizar dinámicamente el comportamiento futuro del precio del petróleo crudo y su volatilidad (Cruz, 2007; Venegas, 2008). Cabe señalar que existen otros métodos alternativos de discretizacion de la ecuación (7) sobre intervalos uniformes e irregulares, conocidos como mallados o conjuntos de nodos con sus relaciones de adyacencias (véase Salt et al., 2016). En cada uno de estos métodos, la transición de (7) a (8) es sensible al tipo de mallado empleado porque el cómputo y la convergencia de los parámetros varían con la geometría del reticulado o la partición del dominio de la ecuación (7). Nosotros elegimos el método descrito por la posibilidad de obtener formas cerradas que faciliten el cómputo de los parámetros por medio de estimaciones estadísticas.

Análisis estadístico de la base de datos

La figura 1 muestra la evolución mensual de los precios en las tres modalidades de petróleo crudo que México exporta al continente Americano (Istmo, Maya y Olmeca) durante el periodo muestral. Las observaciones ascienden a 227 y están expresadas en dólares por barril, según datos obtenidos de la página web de Pemex (ver la sección de referencias). Del total de observaciones, $215(94.74 \%)$ y $221(97.37 \%)$ son usadas, sucesivamente, para calibrar los modelos estadísticos mientras que las restantes son consideradas para computar distintas métricas de errores en el pronóstico, como las sugeridas por Hansen (2005). Así que el análisis de esta sección está referido sólo a las 221 observaciones comprendidas entre enero de 1999 y junio de 2017. 


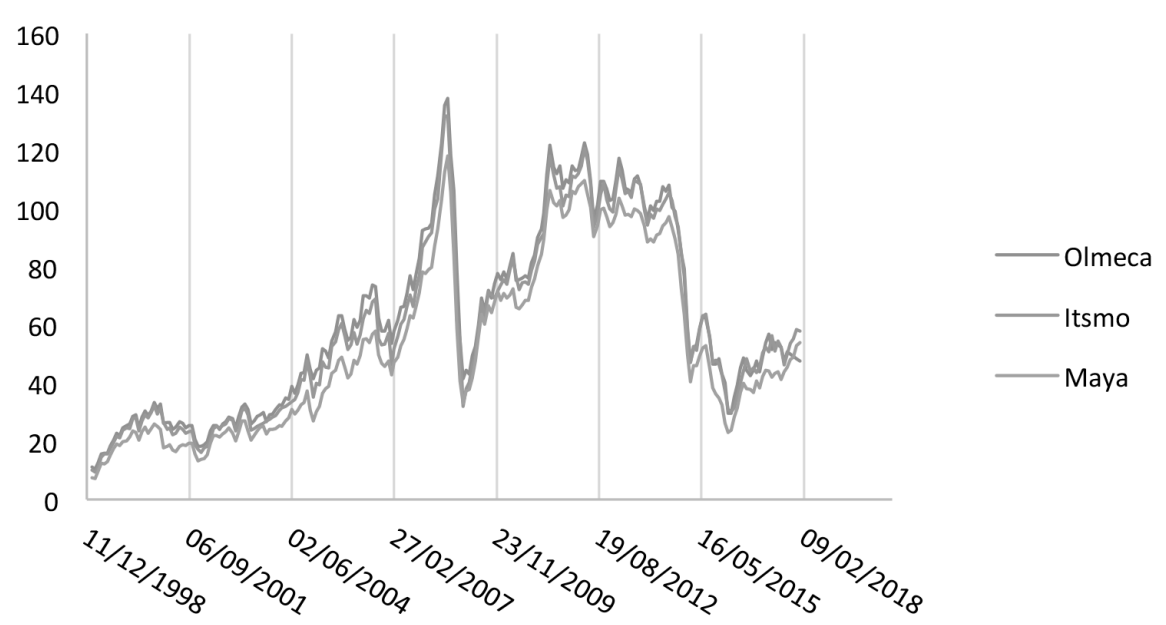

Figura 1. Precios mensuales de las modalidades Olmeca, Istmo y Maya exportadas al continente Americano entre Enero de 1999 y Junio de 2017. Fuente: Elaboración propia con base en datos tomados de: http://www.pemex.com/en/about-pemex/reports-and-publications/Paginas/default. aspx, y http://ebdi.pemex.com/bdi/bdiController.do?action=cuadro\&subAction=applyOptions Nota: En la figura se pueden distinguir seis periodos:[1] Enero de 1999 a julio de 2006; [2] Agosto de 2006 a junio de 2008; [3] Julio de 2008 a diciembre de 2008; [4] Enero de 2009 a abril de 2011; [5] Mayo de 2011 a Enero de 2015 y [6] Febrero de 2015 a junio de 2017.

De acuerdo con la Figura 1, los precios de las tres modalidades se mueven muy cercanamente y con la misma tendencia a lo largo del periodo. En particular, la media de precios más alta la registra la variedad Olmeca (\$61.52) seguida de las del Istmo (\$58.01) y Maya (\$52.42), aunque con una gran volatilidad en los tres casos (32.29\%, 31.65 \% y $30.10 \%$, respectivamente). Este último aspecto se vuelve más evidente si consideramos la volatilidad de los rendimientos para cada una de las modalidades, debido a que ahí queda claro que ésta alcanza un máximo de $24.26 \%$ en el Istmo y un mínimo de $2.62 \%$ en el Maya (ver Tabla 1 ). 
Tabla 1

Rangos de las volatilidades de los rendimientos del petróleo crudo de México en sus tres modalidades.

\begin{tabular}{lccc}
\hline & \multicolumn{3}{c}{ Volatilidad de los rendimientos } \\
\cline { 2 - 4 } & Olmeca & Istmo & Maya \\
\hline Máximo & $18.32 \%$ & $24.26 \%$ & $19.58 \%$ \\
Mínimo & $3.61 \%$ & $2.82 \%$ & $2.62 \%$ \\
Promedio & $8.52 \%$ & $9.09 \%$ & $8.49 \%$ \\
\hline
\end{tabular}

Fuente: Elaboración propia con base en datos tomados de: http://www.pemex.com/en/about-pemex/reports-and-publications/Paginas/default.aspx http://ebdi.pemex.com/bdi/bdiController.do?action=cuadro\&subAction=applyOptions

Nota: La longitud de la ventana móvil es de un año y con ajustes mensuales

Esta volatilidad, sin embargo, no solo es muy irregular por modalidad sino también por sub-periodo de tiempo. La Figura 2 muestra, en efecto, que los rangos de volatilidad crecen a partir de 2008, para después retroceder a su tendencia anterior en febrero de 2010, caer más allá del promedio (8.49\%) entre 2011 y 2014 y, finalmente, rebasar ese valor entre 2015 y 2016. Es decir, tenemos un comportamiento dispar al interior del periodo de observación que obliga a justificar la periodización adoptada.

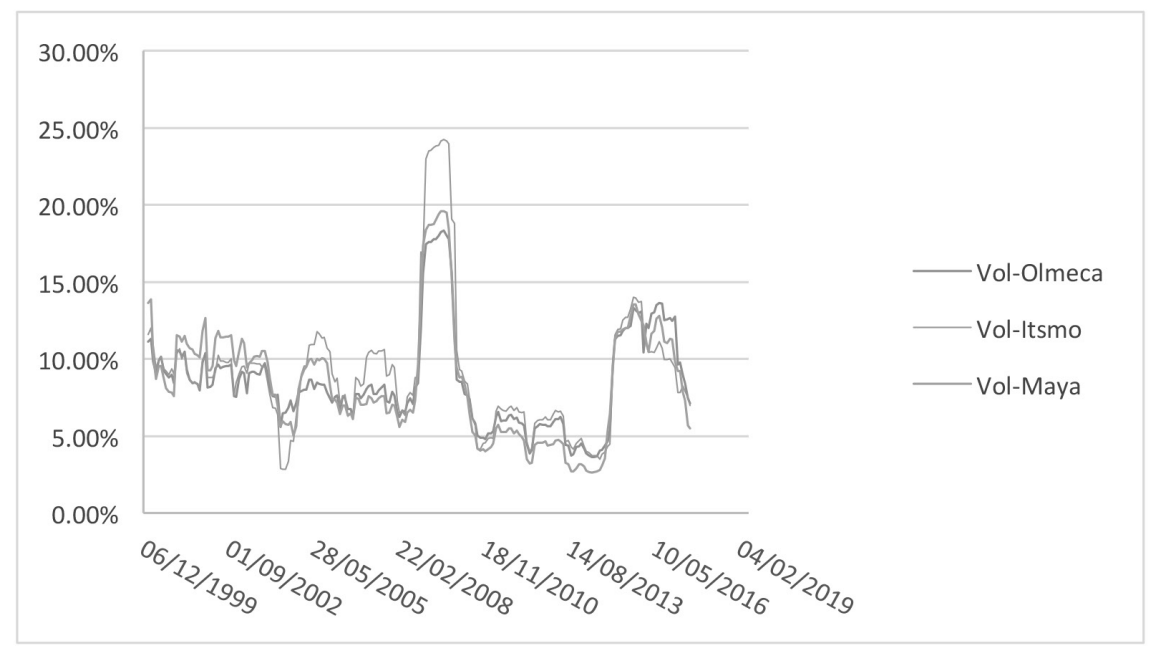

Figura 2. Evolución de la volatilidad anual móvil de los rendimientos del petróleo crudo de México en sus tres modalidades (Enero de1999 a junio de 2017).

Fuente: Elaboración propia con base en datos tomados de: http://www.pemex. com/en/about-pemex/reports-and-publications/Paginas/default.aspx, y 
http://ebdi.pemex.com/bdi/bdiController.do?action=cuadro\&subAction=applyOptions

Nota: El primer dato de la volatilidad de los rendimientos está referido a enero de 2000. La longitud de la ventana móvil es de un año con ajustes mensuales.

Como está ampliamente documentado en la literatura especializada, distintos factores han tenido un peso importante en la volatilidad de los precios internacionales del crudo (véase por ejemplo Baumeister y Lilian 2016). Así tenemos que el alza sostenida de los precios entre 1999 y 2008 (y que abarca los dos primeros sub-períodos de la Figura 1) obedece, en buena medida, al incremento en la demanda de petróleo por países de rápido crecimiento, como China e India. De igual manera, la caída estrepitosa en el precio del barril entre junio y diciembre de 2008 (tercer periodo) se explica por la crisis financiera de 2008-2009; como también se explica el repunte del precio entre enero de 2009 y abril de 2011 por la decisión de la OPEP de mantener una oferta regulada entre sus países miembros (cuarto periodo). El abandono de estas medidas por la OPEP, que mantuvieron estable el precio del barril en niveles por encima de los 100 dólares en los tres años siguientes (quinto periodo), junto con la presencia de un dólar fuerte y el resurgimiento de EEUU como potencia productora de petróleo, dan como resultado una impresionante caída del precio entre julio de 2014 y marzo de 2016 (sexto periodo).

El resultado conjunto de estos vaivenes arroja un marcado patrón de alejamiento de la tendencia, seguido de un regreso no necesariamente duradero al nivel de precios de los periodos anteriores. Esto se puede constatar si se comparan los precios reales del Tabla 2, construidos con base en el índice de precios al productor de EEUU (Enero de 1999=100), para algunos años representativos de los seis sub-períodos. En efecto, en esa Tabla se observa que, para la modalidad Maya, hay un regreso múltiple a su nivel original de precios reales (2005) en los periodos cercanos a diciembre de 2008 , enero de 2015 y junio de 2017 , mientras que para la modalidad Olmeca, dicho regreso es más pronto (enero de 2007) aunque no tan recurrente como en el Maya. Finalmente, en la modalidad Istmo el regreso toma, en promedio, un intervalo de tiempo más largo que en el resto de las modalidades (10 años).

Esta información puede resultar muy útil para explicar diferenciadamente el comportamiento del patrón de reversión a la media en el corto plazo. Basta revisar las estadísticas de la tabla 3 para darnos cuenta de la variedad de medias, volatilidades y distribuciones (normales y no normales) asociadas con cada sub-período y, por tanto, de las grandes posibilidades analíticas que ofrece un estudio por separado. El problema es que dividir el lapso de observación completo en sub-períodos puede acentuar la tendencia ascendente o descendente de la figura 1 y desvanecer el patrón de reversión a la media. 
Tabla 2

Precios reales en dólares por barril para las tres modalidades de petróleo de México en algunas fechas relevantes del periodo comprendido entre Enero de 2005 y Abril de 2017

\begin{tabular}{lccccccc}
\hline \multicolumn{1}{c}{ Fecha } & Olmeca & Istmo & Maya & Fecha & Olmeca & Istmo & Maya \\
\hline Enero 2005 & 43.76 & 33.60 & 25.90 & Mayo 2010 & 53.88 & 54.88 & 47.38 \\
Julio 2006 & 58.26 & 53.75 & 44.98 & Abril 2011 & 80.73 & 78.29 & 70.41 \\
Enero 2007 & 43.31 & 37.27 & 34.49 & Abril 2014 & 68.65 & 64.77 & 59.99 \\
Julio 2008 & 93.58 & 89.49 & 80.26 & Enero 2015 & 32.20 & 32.01 & 27.59 \\
Diciembre 2008 & 31.92 & 24.79 & 25.32 & Febrero 2016 & 18.86 & 21.30 & 16.76 \\
Abril 2010 & 60.81 & 59.62 & 52.08 & Junio 2017 & 35.19 & 35.14 & 27.71 \\
\hline
\end{tabular}

Fuente: elaboración propia con datos de Pemex http://www.pemex.com/en/about-pemex/reports-and-publications/ Paginas/default.aspx y de las estadísticas de Producer Price Index (Total Manufacturing Index) del US Bureau of Labor Statistics en https://www.bls.gov/ppi/

Nota: los precios nominales fueron deflactados tras hacer un cambio de base de Dic de 1984 a enero de 1999 en la serie desplegada por https://fred.stlouisfed.org/series/PCUOMFGOMFG.

Para empezar, no hay que olvidar que las medidas de persistencia de la reversión a la media requieren que los procesos auto-regresivos no sean de raíz unitaria o, de igual manera, que los shocks sobre las series de precios no sean permanentes. Una periodización que comprenda los meses de enero de 1999 a junio de 2008 o los dos primeros sub-períodos de la Figura 1, por ejemplo, carece de sentido, porque es evidente que en ese lapso no hay ningún regreso visible de los precios reales a un hipotético nivel propuesto y, por lo tanto, es altamente probable que las medidas de persistencia de reversión a la media (como las funciones de impulso respuesta) no tengan significado alguno. Del mismo modo, tomar por separado otra gran división, como la que incluye las alzas y bajas abruptas del periodo julio de 2008- junio de 2017 (o de los cuatro restantes sub-periodos de la Figura 1), puede resultar inadecuado, porque es muy seguro que los shocks en los precios con una larga vida media de sobrevivencia no hayan sido todavía absorbidos por la tendencia. El análisis de los patrones de reversión requiere periodos muy largos para observar el agotamiento de los efectos de los shocks sobre los precios, por lo que cualquier granulación excesiva de una periodización es riesgosa, sobre todo si las series, como la de la Figura 1, exhibe distintos regresos en los niveles de precios (ver Tabla 2) y una tendencia suavizada al final que indica la estabilización de los shocks. El análisis de los sub-periodos resaltados en la Figura 1 debe, pues, incluir otros métodos estadísticos idóneos para muestras pequeñas que complementen al entendimiento del patrón de la reversión a la media, no que lo sustituyan.

El análisis estadístico se concentra en la modalidad del crudo Maya y en el mercado Americano por ser el producto y la región que mayor importancia tienen en las exportaciones 
mexicanas de crudo al exterior (80.29\%). No obstante que la proporción de ese mercado ha ido disminuyendo significativamente en las ventas mexicanas de crudo (al pasar del 81.2 por ciento en 1999 al 52\% en 2017) su importancia en el periodo de estudio es mayor que la de cualquier otra modalidad, por lo que las conclusiones aquí obtenidas no pierden generalidad.

Tabla 3

Estadísticas descriptivas de las series de precios del petróleo crudo en México para las tres modalidades en seis sub-periodos de tiempo

\begin{tabular}{|c|c|c|c|c|c|c|c|}
\hline \multirow[b]{2}{*}{$\begin{array}{l}\text { P e tróle o } \\
\text { crudo }\end{array}$} & \multicolumn{6}{|c|}{ Enero de 1999 a julio de 2006} & \multirow[b]{2}{*}{ Probabilidad } \\
\hline & Media & Varianza & $\begin{array}{l}\text { Volatilidad de } \\
\text { los precios }\end{array}$ & Sesgo & Curtosis & Jarque Bera & \\
\hline Olmeca & 33.9229 & 220.7480 & 14.8576 & 0.9985 & 3.1473 & 15.2036 & 0.0005 \\
\hline Istmo & 31.7375 & 186.8394 & 13.6689 & 0.9680 & 3.1170 & 14.2633 & 0.0008 \\
\hline Maya & 26.4386 & & 11.3985 & 0.9952 & 3.3629 & 15.5216 & 0.0004 \\
\hline \multicolumn{8}{|c|}{ Agosto de 2006 a junio de 2008} \\
\hline Olmeca & 80.1745 & 496.7527 & 22.2880 & 0.9139 & 2.9832 & 3.2020 & 0.2017 \\
\hline Istmo & 75.0514 & 518.4428 & 22.7693 & 0.9110 & 3.0350 & 3.1825 & 0.2036 \\
\hline Maya & 66.3336 & 379.6535 & 19.4847 & 0.7767 & 2.6896 & 2.4046 & 0.3005 \\
\hline \multicolumn{8}{|c|}{ Julio de 2008 a diciembre de 2008} \\
\hline Olmeca & 89.3832 & 1406.8673 & 37.5082 & -0.0523 & 1.5606 & 0.5207 & 0.7707 \\
\hline Istmo & 82.3397 & 1480.4318 & 38.4764 & -0.0143 & 1.5292 & 0.5410 & 0.7630 \\
\hline Maya & 73.2120 & 1208.7860 & 34.7676 & 0.1083 & 1.4631 & 0.6022 & 0.7399 \\
\hline \multicolumn{8}{|c|}{ Enero de 2009 a abril de 2011} \\
\hline Olmeca & 76.0438 & 309.5819 & 17.5949 & 0.3721 & 3.7626 & 1.3245 & 0.5157 \\
\hline Istmo & 73.4325 & 307.9639 & 17.5489 & 0.1692 & 3.7879 & 0.8578 & 0.6512 \\
\hline Maya & 68.1441 & 246.4429 & 15.6985 & 0.1377 & 3.6544 & 0.5882 & 0.7452 \\
\hline \multicolumn{8}{|c|}{ Mayo de 2011 a enero de 2015} \\
\hline Olmeca & 103.8125 & 197.6060 & 14.0572 & -2.3535 & 9.3587 & 117.3552 & 0.0000 \\
\hline Istmo & 101.4669 & & 13.1273 & -2.3579 & 9.8224 & 129.0048 & 0.0000 \\
\hline Maya & 98.8672 & & 13.5287 & -2.3836 & 9.0753 & 111.8153 & 0.0000 \\
\hline \multicolumn{8}{|c|}{ Febrero de 2015 a junio de 2017} \\
\hline Olmeca & 47.9396 & 68.9507 & 8.3037 & -0.3857 & 2.9568 & 0.7213 & 0.6972 \\
\hline Istmo & 47.2895 & 75.6678 & 8.6987 & -0.2681 & 2.6646 & 0.7853 & 0.7853 \\
\hline Maya & 39.1513 & 58.2755 & 7.6338 & -0.4103 & 2.7485 & 0.8901 & 0.6408 \\
\hline
\end{tabular}

Fuente: Elaboración propia con base en datos de http://www.pemex.com/en/about-pemex/reports-and-publications/ Paginas/default.aspx 


\section{Resultados}

La segunda etapa de la metodología consiste en estimar los parámetros de las ecuaciones (8) y (9) $a_{x}, b_{x}$ y $\hat{P}(x, t)$, seleccionar el mejor ajuste estadístico, aplicar la prueba de Andrews, utilizar algunas versiones muestrales de funciones de pérdidas y hacer algunas métricas de error de pronóstico de los modelos construidos con el $94.74 \%$ y $97.37 \%$ de la muestra. Entre estas métricas consideramos las que ya son comunes en la literatura especializada como son: el Error Cuadrático Medio (MSE), la Raíz cuadrada del Error Cuadrático Medio (RMSE), la Desviación Media Absoluta (MAD) y el Error Promedio Absoluto Porcentual (MAPE) (véase Hansen y Lunde, 2005; Safari y Davallou,2018).

Con respecto a la prueba de Andrews es importante mencionar que es una estimación insesgada de la mediana de un proceso auto regresivo de primer orden y de raíz unitaria (AR/ RU), usada para determinar la persistencia de los shocks sobre las series de precios. Si la serie tiene raíz unitaria, la función de impulso-respuesta (FIR) empleada en la prueba nunca se desvanecerá o, dicho de otra manera, el shock sobre la serie de precios será permanente, mientras que si la serie es estacionaria con tendencia, la FIR desaparecerá en un tiempo finito y los shocks serán temporales. La FIR es definida como:

$$
\operatorname{FIR}(\tau)=\alpha^{\tau} \text { para } \tau=0,1,2, \ldots(10)
$$

donde a es el estimador insesgado de la mediana del parametro autoregersivo y $\tau$ es el horizonte de tiempo donde se mide el shock. Otras dos medidas escalares de persistencia ligadas con la FIR son la funcion acumulada de impulso respuesta (AIR) y la vida media de una unidad de shock (MVS). Ambas las podemos expresar de la siguiente manera:

$$
\begin{gathered}
A I R=\sum_{t=0}^{\infty} F I R(\tau)=(1-\alpha)^{-1} \\
V M S=A B S\left(\frac{\ln \left(\frac{1}{2}\right)}{\ln \alpha}\right)(12)
\end{gathered}
$$

La ventaja de usar la prueba de Andrews por encima de las medidas estándares de raiz unitaria de Dickey-Fuller o Philipps-Perron radica en su capacidad de corregir los sesgos introducidos por las asimetrías en las distribuciones de los estimadores de los parámetros 
autoregresivos, que no son contempladas por esas medidas tradicionales. Las distribuciones por lo general son sesgadas hacia la izquierda y presentan colas pesadas, como en efecto sucede con las series de precios del petroleo aquí consideradas, y eso provoca que la mediana sea mejor estimador que la media como medida de tendencia central en la estimaciones de minimos cuadrados de los modelos AR/RU. Adicionalmente, la prueba combina la propiedad de insesgadez con el uso de estimaciones puntuales y por intervalos, que reducen la probabilidad de cometer errores tipo 1 y II en la determinacion de la persistencia de los shocks sobre las series de precios (Cashin et al., 2000: Andrews 1993).

En la estimacion de los modelos $A R(1)$ consideramos que los valores de $\hat{P}(x, t)$ que tienden $a b_{x}$ son las proxies de los precios racionales $P_{t}^{*}$ y los valores de $P_{t}$ son los precios nominales deflactados por el índice de los precios al productor de EE UU con base en Enero de 1999.

\section{El modelo de ajuste para el periodo que incluye al $97.37 \%$ de la muestra}

Al considerar la serie de precios entre enero de 1999 y junio de 2017 observamos que el modelo de ajuste de la ecuación (8) desplegado en (13), si bien presenta un $\mathrm{R}^{2}$ muy alto $(97.95 \%)$ está afectado por auto correlación serial y heteroscedasticidad. En efecto, al computar las pruebas de Durbin-Watson (DW), Breusch-Godfrey (BG) y las de White, tenemos que las hipótesis nulas de no auto correlación serial y homoscedasticidad son rechazadas consistentemente en (13). En concreto, para la prueba de auto correlación serial se encuentra que $B G=55.08826>\chi^{2}=5.99$ mientras que para la de homoscedasticidad el estadístico arroja el valor, con $T R_{\text {errores }}^{2}=11.0217>\chi^{2}=5.99$, con $T=221$ y DW=1.0132.

$$
y_{t+1, \text { Maya }}=1.8119 y_{t ; \text { Maya }}^{-1}+0.9919 y_{t ; \text { Maya }}+u_{t} .
$$

Para subsanar estos problemas aplicamos, inicialmente, la corrección $\rho=1-\mathrm{DW} / 2=0.4933775$ a cada una de las variables de la ecuación (13) para, luego, extraer su primera diferencia. El nuevo modelo resultante en (14) es, de acuerdo con la prueba de mínima varianza de White-Huber, no heteroscedastico. A diferencia de la prueba de White, que utiliza una matriz de correlación y el termino cruzado de las variable explicativas para rechazar la hipótesis nula de no heteroscedasticidad, la prueba de White-Huber utiliza dicha matriz para minimizar la varianza de los errores en el modelo utilizado. En este sentido es más completa que la prueba de White. De aquí que tras obtener un valor $p$ de 0.0843 y un estadístico $\mathrm{t}=-1.7345$ en el coeficiente del término cruzado, éste y el coeficiente de las variables explicativas al cuadrado no resultan estadísticamente significativos en la varianza de los errores, por lo que la varianza de los residuales al cuadrado registra un valor igual a cero. Otros estadísticos de (14) confirman la no autocorrelación serial: $B G=0.3750<\chi^{2}=5.99$ y DW=1.9173, además de mostrar un $\mathrm{R}^{2}=94.03 \%$ muy alto. 


$$
\Delta y_{t+1, \text { Maya }, r t}=3.7557 \Delta y_{t ; \text { Maya }}^{-1}+0.9810 \Delta y_{t ; \text { Maya }, r t}+u_{t} .
$$

Los dos parámetros estimados en (14) no son necesariamente puntuales, sino que pueden también incluirse en intervalos con diferentes niveles de confianza, tal como se muestra en la Tabla 4. Así tenemos, por ejemplo, que el valor del estimador insesgado de la mediana de $\Delta y_{t ; \text { Maya,rt }}$ puede estar contenido dentro de los valores mínimos (965313) y máximos (0.996754) de un intervalo del 95\% o, dependiendo del cuantil de ${ }^{a}$ y del tamaño de muestra, dentro de intervalos acotados por la izquierda o la derecha, como veremos a continuación.

Tabla 4

Intervalo de los valores de los parámetros de (14) con diferentes niveles de confianza

\begin{tabular}{lllllll}
\hline \multirow{2}{*}{ Coeficiente } & \multicolumn{2}{c}{$90 \%$} & \multicolumn{2}{c}{$95 \%$} & \multicolumn{2}{c}{$99 \%$} \\
\cline { 2 - 7 } & mínimo & máximo & mínimo & máximo & mínimo & máximo \\
\hline 3.756792 & 1.587996 & 5.925588 & 0.169145 & 6.344440 & 0.345121 & 7.168464 \\
0.981034 & 0.967858 & 0.994210 & 0.965313 & 0.996754 & 0.960307 & 1.001760 \\
\hline
\end{tabular}

Fuente: Elaboración propia.

Un aspecto importante es que (14) nos permite calcular rápidamente los valores de los parámetros $\widehat{a_{x}}=0.037932$ y $\widehat{b_{x}}=37.825686$ una vez obtenido $\hat{\beta}_{1}$ y $\hat{\beta}_{2}$ (ver ecuación 8), así como la serie de precios de la ecuación (9), desplegada en la figura 4. Ahí se puede apreciar que $P_{t}^{*}$ se mueve hacia una media $\widehat{b_{x}}$ de 37.8257 dólares/barril de largo plazo con velocidad $\widehat{a_{x}}$ de 0.0379 dólares/barril/mes. Esta tendencia a la reversión a la media es confirmada por la prueba de Andrews $P_{\alpha}=\widehat{\alpha_{m c o}}=q_{p}(\alpha)=p=P_{0.98}(=0.98103 \leq 0.998)$ en la que se aprecia que el estimador insesgado de la mediana del parámetro auto regresivo está contenido en el intervalo de confianza del 95\% y, por lo tanto está medido con bastante precisión. Para ver con más claridad este punto observemos que por (10) la FIR tenderá a desvanecer en el tiempo a medida que $\tau$ crece, pues en ese caso $\lim _{\tau \rightarrow \infty} \alpha^{\tau}=0$ porque $\alpha=0.98103$. Entonces, si bien es cierto que cualquier shock unitario a los precios del crudo Maya provoca un desplazamiento significativo en su nivel general de 52.71 unidades (por la ecuación 11) durante este periodo, también es cierto que en una cantidad finita de meses, 36.18 meses (por la ecuación 12), la mitad del impulso respuesta de ese shock tenderá a disiparse. 


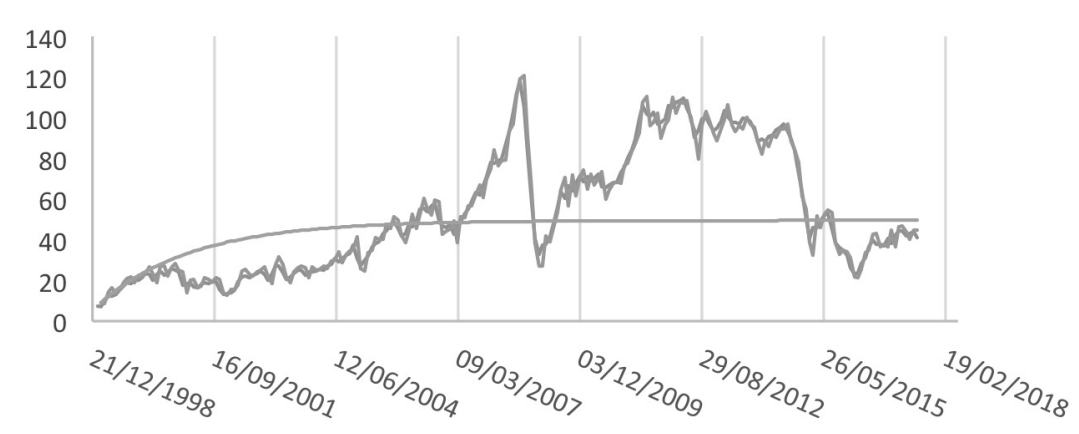

Figura 3. Dinámica de los precios del petróleo crudo Maya en México entre enero de 1999 y junio de 2017 Fuente: Elaboración propia.

El modelo de ajuste para el periodo que incluye el $94.74 \%$ de la muestra

Para el periodo más reducido que incluye los 216 meses entre enero de 1999 y diciembre de 2016 el modelo (15) presenta un mejor ajuste $\left(R^{2}=97.96\right)$ que (13) porque sus estadísticos de prueba no rechazan las hipótesis nula de homoscedasticidad $\left(T R_{\text {errores }}^{2}=3.376541<\chi^{2}=5.99 ; \operatorname{con} T=216\right)$ ni de no auto correlación serial $(\mathrm{DW}=1.990609)$.

$$
y_{t+1, \text { May }}=1.817518 y_{t ; \text { Maya }}^{-1}+0.991987 y_{t ; \text { Maya }}+u_{t}
$$

Asimismo, las estimaciones puntuales $\left(P_{0.99}(=0.991987 \leq 0.998)=0.05\right)$ y de intervalo para los coeficientes de los parámetros auto regresivos son insesgadas respecto a la mediana para distintos niveles de confianza (ver Tabla 5), con lo que se confirma que el proceso no es de raíz unitaria.

Tabla 5

Intervalo de valores de los parámetros de (15) con diferentes niveles de confianza

\begin{tabular}{llclccc}
\hline \multirow{2}{*}{ Coeficiente } & \multicolumn{3}{c}{$90 \%$} & \multicolumn{2}{c}{$95 \%$} & \multicolumn{2}{c}{$99 \%$} \\
\cline { 2 - 7 } & mínimo & máximo & mínimo & máximo & mínimo & máximo \\
\hline 1.817518 & 0.441626 & 3.193410 & 0.175855 & 3.459182 & -0.347088 & 3.982124 \\
0.991987 & 0.983900 & 1.000073 & 0.982338 & 1.001635 & 0.979265 & 1.004708 \\
\hline
\end{tabular}

Fuente: Elaboración propia. 
De lo anterior, se tienen que el proceso auto regresivo converge a la media de largo plazo $\widehat{b_{r}}=38.7484784$ con una velocidad de ajuste $\widehat{a_{x}}=0.016026$ a través de la trayectoria de $\hat{P}(x, t)=\left(\frac{\sqrt{x}}{2}\right)^{2}$, como se observa en la Figura 4. Esto quiere decir que la FIR tiende a desaparecer en un periodo finito en virtud de que $\lim _{\tau \rightarrow \infty} \alpha^{\tau}=0$ porque $\alpha=0.98103$. Concretamente la tendencia tiende a desplazarse 123.41 unidades tras shocks en los precios (por la ecuación 11) para, después, recuperar la mitad de su nivel original en 86.08 meses (por la ecuación 12).

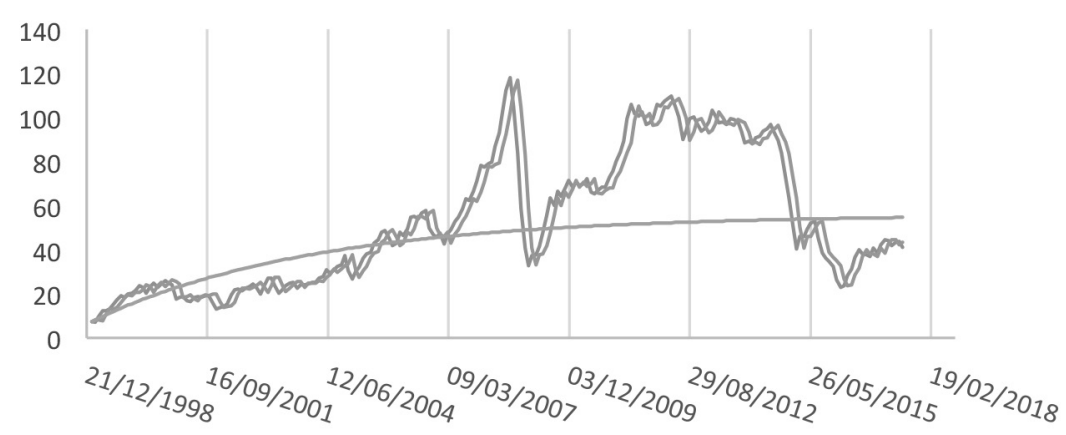

Figura 4. Dinámica de los precios del petróleo crudo Maya en México para el periodo comprendido entre enero de 1999 y diciembre de 2016.

Fuente: Elaboración propia.

\section{Pronósticos}

El comportamiento de los precios generados por la tendencia a la reversión de la media muestra un proceso aun inacabado en las Figuras 3 y 4 . Las medias de sus precios racionales es un límite al cual converge la serie de precios reales en caso de que se mantenga el patrón descrito por los datos entre enero de 1999 y junio de 2017, no un precio dado para cumplirse en el periodo muestral (pues hay todavía shocks no absorbidos por la tendencia). Por esa razón, es explicable que las figuras revelen niveles de precios superiores a sus medias en las fechas recientes, como queda claro en los siguientes pronósticos que hacemos para ventanas de seis (julio-diciembre de 2017) y doce meses (enero-diciembre de 2017), en los que el precio ronda un valor superior a los 50 dólares por barril.

En las Tablas 6 y 7 se muestran los precios observados y pronosticados para esas ventanas con diferentes proporciones muestrales (97.37\% y 94.74\%). En ambas tablas se puede apreciar que las diferencias entre ambas series disminuyen sensiblemente al término del periodo pronosticado. En particular destaca el hecho que la especificación (14) muestra una convergencia más acelerada que la de (15), como lo constatan los menores valores de MSE, 
RMSE, MAD y MAPE en la Tabla 8 para el pronóstico que incluye el 97.37\% de la muestra.

De aquí que entre más datos recientes incorpore el modelo AR o, dicho de otro modo, entre mayor número de shocks de largo plazo absorba la tendencia, más preciso es el pronóstico y menor la variabilidad existente entre los datos pronosticados y observados. Por este motivo, obviamos la práctica extendida en alguna parte de la literatura de entrenar los modelos con menores porcentajes de la muestra que los aquí sugeridos y evaluar el pronóstico dentro y fuera de la muestra con las proporciones restantes. El procedimiento aquí empleado para evaluar los pronósticos del modelo ajusta, pues, los porcentajes al tamaño de la muestra y a la naturaleza de la información tratada y toma las métricas de error como una medida de referencia global de los desempeños de los modelos.

Tabla 6

Pronóstico de los precios del petróleo crudo mediante el modelo de reversión a la media (14)

\begin{tabular}{cccc}
\hline & & \multicolumn{2}{c}{ Maya } \\
Porcentaje de la muestra & 2017 & Observado & Pronóstico \\
& Julio & 43.877714 & 54.659868 \\
& Agosto & 45.554842 & 54.680660 \\
& Septiembre & 48.162395 & 54.701115 \\
& Octubre & 48.891212 & 54.721238 \\
& Noviembre & 52.824919 & 54.741036 \\
& Diciembre & 53.871941 & 54.760513 \\
\hline
\end{tabular}

Fuente: Elaboración propia.

Tabla 7

Pronóstico de los precios del petróleo mediante el modelo de reversión a la media (15)

\begin{tabular}{ccccccc}
\hline $\begin{array}{c}\text { Porcentaje } \\
\text { de la mues- } \\
\text { tra }\end{array}$ & \multicolumn{5}{c}{ Maya } \\
& 2017 & Observado & Pronóstico & 2017 & Observado & Pronóstico \\
& Enero & 44.500073 & 55.213072 & Julio & 43.877714 & 55.350944 \\
& Febrero & 44.174861 & 55.236989 & Agosto & 45.554842 & 55.372651 \\
& Marzo & 41.932469 & 55.260522 & Septiembre & 48.162395 & 55.394010 \\
$94.74 \%$ & Abril & 43.221241 & 55.283678 & Octubre & 48.891212 & 55.415026 \\
& Mayo & 43.850742 & 55.306463 & Noviembre & 52.824919 & 55.435706 \\
& Junio & 41.151130 & 55.328883 & Diciembre & 53.871941 & 55.456054 \\
\hline
\end{tabular}

Fuente: Elaboración propia. 
Tabla 8

Criterios de evaluación de la robustez de los dos modelo de reversión a la media

\begin{tabular}{ccccccc}
\hline \multirow{2}{*}{$\begin{array}{c}\text { Porcentaje de la } \\
\text { muestra }\end{array}$} & Periodo (2017) & Petróleo & MSE & RMSE & MAD & MAPE \\
& De Julio a Diciembre & Maya & 39.4920 & 6.2843 & 5.1911 & $11.07 \%$ \\
$97.37 \%$ & De enero a Diciembre & Maya & 102.0606 & 10.1025 & 9.3367 & $21.11 \%$ \\
$94.74 \%$ & &
\end{tabular}

Fuente: Elaboración propia.

\section{Conclusiones}

En este trabajo ofrecemos evidencia de un patrón de reversión a la media de largo plazo en la serie de precios del petróleo crudo Maya exportado por México al continente Americano entre enero de 1999 y junio de 2017. Los precios reales de esta modalidad guardan en este periodo patrones pronosticables en el corto plazo y, por tanto, no siguen el comportamiento de una caminata aleatoria con incrementos independientes tal como lo supone la HME.

Los dos modelos AR (I) aquí desarrollados muestran una media mensual de largo de plazo de 37.8 (con $97.37 \%$ de la muestra) y 38.7 (94.7\% de la muestra) dólares por barril con diferentes velocidades de convergencia (menor en el segundo caso). Esto quiere decir que, ante un shock en los precios, las FIR de sus parámetros auto regresivos tenderán a cero pero con distinta rapidez: en el modelo con mayor proporción muestral, el regreso a su nivel original tomara menos de la mitad de tiempo que con el de menor proporción muestral (36.18 versus 86.08 meses), debido a que los desplazamientos por unidades provocados por los shocks son más duraderos en el segundo que en el primero (123.4 versus 52.71). A causa de estas diferencias, las predicciones en ambos casos también divergen, pues aunque los dos modelos arrojan resultados muy aceptables entre los precios observados y los pronosticados, el que incluye mayor proporción muestral presenta menores errores de pronóstico.

En cualquier caso, la conclusión no cambia: los precios reales exhiben un patrón de reversión a la media y queda como tarea pendiente para estudios posteriores determinar cuáles son los factores que permiten caracterizar el pronóstico de las series en el corto plazo. En esa tarea hay que considerar la periodización sugerida en la Figura 1 y los determinantes esbozados para cada sub-periodo.

\section{Referencias}

Andrews, D. (1993). Exactly median-unbiased estimation of first order autoregressive/Unit root models. Econometrica, Vol. 61 (1), 139-165. https://doi.org/10.2307/2951781 
Baumeister, C., y Kilian, L. (2016). Forty years of oil price fluctuations: Why the oil price may still surprise us. The Journal of Economic Perspectives, Vol 30 (1), 139-160. http:// dx.doi.org/10.2139/ssrn.2714319

Bermúdez, N. Bermúdez, E, y Saucedo, E. (2018). The relationship between oil prices, the stock market and exchange rate: Evidence from Mexico. North American Journal of Economics and Finance, Vol.45, 266-275. https://doi.org/10.1016/j.najef.2018.03.006

Cashin, P., Liang, H. y McDermott, J. (2000). How persistent are shocks to world commodity prices? IMF Staff Papers, Vol.47 (2), 177-217. http://dx.doi. org/10.5089/9781451850284.001

Cruz A. F. (2007). Valor en riesgo de bonos cupón cero en el mercado mexicano con los modelos Vasicek y CIR: Simulación Monte Carlo con saltos de Poisson y valores extremos, Tesis de Doctorado, ITESM.

Choi, K., y Hammoudeh, S. (2009). Long memory in oil and refined products markets. The Energy Journal, Vol. 30 (2), 97-116. http://dx.doi.org/10.5547/ISSN0195-6574-EJVol30-No2-5

Cox, J., Ingersoll, E. y Ross, S. (1985). A Theory of the Term Structure of Interest Rates. Econometrica, 53(2), 385-408. https://doi.org/10.1142/9789812701022_0005

Hamilton, J. (2009). Understanding oil prices. The Energy Journal, Vol. 30 (2), 179-206. https://doi.org/10.5547/ISSN0195-6574-EJ-Vol30-No2-9

Hammoudeh, S. (1996). Mean reversion and zone readjustments. Southern Economic Journal, Vol. 62 (4), 916-929. https://doi.org/10.2307/1060937

Hansen, P. (2005). A test for superior predictive ability. Journal of Business \& Economic Statistics, 23, 365-380. http://dx.doi.org/10.1198/073500105000000063

Hansen, P. R. y Lunde, A. (2005). A forecast comparison of volatility models: Does anything beat a GARCH (1,1)?. Journal of Applied Econometrics, 20, 873-889. http://dx .doi. org/10.1002/jae.800

http://www.pemex.com/en/about-pemex/reports-and-publications/Paginas/default.aspx http://ebdi.pemex .com/bdi/bdiController.do?action=cuadro\&subAction=applyOptions

Li, J. y Thompson, H. (2010). A note on the oil price trend and Garch shocks. The Energy Journal, Vol.31 (3), 159-165: http://mpra.ub.uni-muenchen.de/20654/

Safari, A., y Davallou, M. (2018). Oil price forecasting using a hybrid model. Energy, 148, 49-58.

https://doi.org/10.1016/energy.2018.01.007

Salt et al. (2016)- Control Automático, Tiempo Continuo y Tiempo Discreto. Serie de Textos Universitarios. Ed. Reverte, S. A. ISBN: 978-84-291-4753-7.

Shiller, R. (2003). Efficient markets theory to behavioral finance. The Journal of Economic Perspectives, Vol.17 (1), 83-104. https://doi.org/10.1257/089533003321164967

Vasicek, O. A. (1977). An Equilibrium Characterization of the Term Structure. Journal of Financial Economics, 5, 177-188. https://doi.org/10.1016/0304-405X(77)90016-2 
Valdés, A., Armenta, L., y Duran, R. (2016). A copula T-Garch approach of conditional dependence between oil price and stock market index: The case of Mexico. Estudios Económicos, Vol. 31 (1), 47-63. Disponible en: https://estudioseconomicos.colmex.mx/ archivo/EstudiosEconomicos2016/47-63.pdf

Venegas M. F. (2008). Riesgos Financieros y Económicos. Cengage Learning. Segunda edición. ISBN-13: 9789708300087 ; eBook: 9786074813692 